\title{
Isolation and Characterization of New Tetrasaccharides from Cottonseed
}

\author{
Koji Kato, Masatoshi Abe, Katsuaki Ishiguro \\ and Yoshimitsu UeNo \\ Department of Agricultural Chemistry, Faculty of Agriculture, \\ Gifu University, Kakamigahara, Gifu 504, Japan \\ Received August 8, 1978
}

\begin{abstract}
Two kinds of new tetrasaccharide were isolated from cottonseed and their structures were established as $1^{\mathrm{F}}-\beta$-D-fructofuranosylraffinose; $O-\alpha$-D-galactopyranosyl-( $\left.1 \rightarrow 6\right)-O-\alpha$-D-gluco-

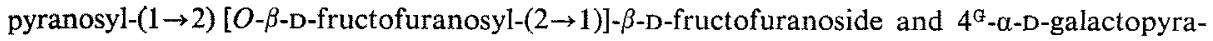
nosylraffinose; $O-\alpha$-D-galactopyranosyl-( $1 \rightarrow 6)[O-\alpha$-D-galactopyranosyl] $(1 \rightarrow 4)]-O-\alpha$-D-glucopyranosyl-(1 $\rightarrow 2)-\beta$-D-fructofuranoside, respectively.
\end{abstract}

Galactosyl- and fructosylsucroses are widely distributed in nature, particularly in planttissues, ${ }^{1)}$ and some of their structures are related to a specificity of corresponding carbohydrases, $\alpha$-D-galactosidase and invertase, in the tissues, respectively. We obtained two kinds of new tetrasaccharide from cottonseed during the course of studies on oligosaccharides in oil-seeds. They were eluted with almost the same retention volumes on a carbon column chromatography, and located between raffinose and stachyose on paper chromatograms with identical $R f$ values. However, they could be separated from each other by a Dowex-1 (borate form) column chromatography and characterized as shown in Fig. 6, by enzymic hydrolyses and methylation studies, respectively.

\section{MATERIALS AND METHODS}

Material. Cottonseed meal (after oil extraction) was used for extraction of oligosaccharides instead of live cottonseed. It was kindly supplied from Gifuken Kumiai Feed Co., Ltd., Gifu, Japan.

General methods. All evaporations were carried out under reduced pressure at $40 \sim 45^{\circ} \mathrm{C}$. The melting point is uncorrected. Specific rotations were determined at $20 \pm 5^{\circ} \mathrm{C}$. Paper chromatography(ppc) was performed by the multiple ascending method on Toyo filter paper No. 51 (for qualitative and quantitative analysis) and No. 525 (for preparation) with the following solvents: (A) pyridine-1-butanol-water (4:6: $3, v / v)^{2)}$ and (B) ethyl acetate-pyridine-water $(10: 4: 3$, $\mathrm{v} / \mathrm{v})^{\left.{ }^{3}\right)}$ Unless otherwise noted, the former solvent was used. Paper electrophoresis was performed on Toyo filter paper No. 51 at $15 \mathrm{v} / \mathrm{cm}$ for $3 \mathrm{hr}$ in $0.1 \mathrm{M}$ borate buffer. ${ }^{4)}$ The position of the compound on the chromatogram was determined by spraying with aniline hydrogen phthalate (AHP), ${ }^{57}$ urea-acid (UA) ${ }^{8}$ ) or alkaline silver nitrate. ${ }^{7)}$ Thin-layer chromatography (tlc) was performed on $0.25 \mathrm{~mm}$ layer of silica gel $G$ (a product of ICN Woelm Laboratories, Inc.) with the solvents of chloroform-methanol. The chromatograms were developed by spraying with $50 \%$ sulfuric acid, AHP or UA. Alditol acetates derived from the oligosaccharides were analyzed with a combined gas chromatograph-mass spectrometer (GC-MS), Hitachi M-52. The compound was separated on a $2 \% \mathrm{OV}_{-1}$ glass column $(0.3 \times 100 \mathrm{~cm})$ at $160^{\circ} \mathrm{C}$ and identified by the spectrum recorded at $20 \mathrm{eV}$ of ionizing potential.

\section{RESULTS}

\section{Extraction of the oligosaccharides}

Cottonseed meal $(4.5 \mathrm{~kg})$ was extracted with $80 \%$ methanol at room temperature. The extraction was repeated until $\alpha$-naphthol reaction of the supernatant became faint. Calcium hydroxide was added to the supernatant and the whole mixture was allowed to stand at room temperature overnight in order to precipitate the impurities such as proteins and phenolic substances completely. The supernatant was neutralized with dil. sulfuric acid and concentrated to about one tenth of the original volume. Insoluble materials occurred during concentration were removed by filtration, and the filtrate was concentrated to a syrup after deionization with Amberlite IR-120 and IRA-410, yield 
$370 \mathrm{~g}$. An examination of the syrup by ppc revealed the presence of $\mathrm{D}$-fructose $(2.3 \%)$, sucrose $(23.6 \%)$, raffinose $(38.4 \%)$, stachyose $(16.3 \%)$, verbascose $(6.6 \%)$ and an unknown sugar $(12.9 \%)$. The ratio of these sugars was determined by phenol-sulfuric acid method ${ }^{8}$ ? after being separated by ppc.

\section{Fractionation of the oligosaccharides}

When the syrup ( $370 \mathrm{~g}$ ) was dissolved in a minimum amount of water and kept at $4{ }^{\circ} \mathrm{C}$ for a few days, crystalline raffinose $(50 \mathrm{~g})$ was obtained which was removed by filtration. The filtrate ( $300 \mathrm{~g}$ as syrup) was placed on a top of a carbon column $(13 \times 75 \mathrm{~cm})$ and eluted successively with water and aqueous 1-pro-

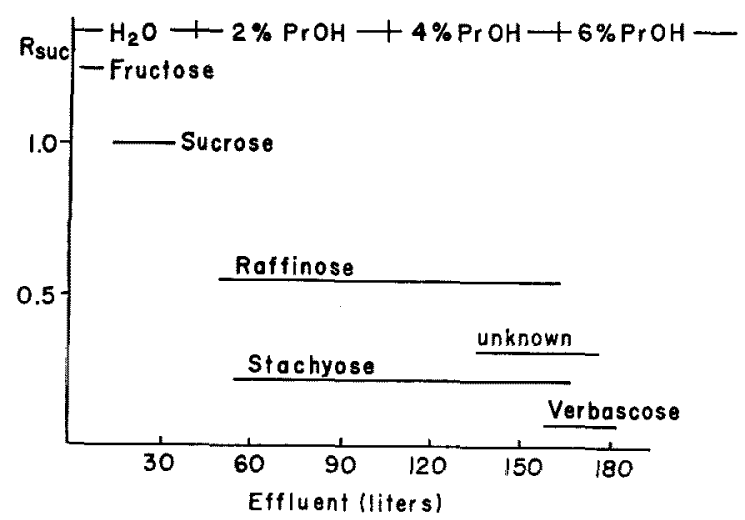

Fig, 1. Carbon-column and Paper-chromatography of $80 \%$ Methanol-extract of Cottonseed Meal.

Paper chromatogram was developed with urea-acid. panol by stepwise elution. Sugar composition of the effluent was examined on ppc developed with UA (Fig. 1). A part (40 mg as syrup) of the $4 \sim 6 \%$ propanol-eluted fraction containing the unknown sugar was dissolved in a small amount of water and then subjected to a Dowex-1 $(200 \sim 400$ mesh, $\times 8$, borate form) column $(12 \times 100 \mathrm{~mm})$ chromatography ${ }^{9}$ by gradient elution with $0 \sim 0.025 \mathrm{M}$ potassium borate at a flow rate of $0.9 \mathrm{ml} / \mathrm{min}$. The carbohydrate-content of the effluent was determined by anthrone-sulfuric acid method. ${ }^{10)}$ The result is shown in Fig. 2. The sugars corresponding to each peak were identified by ppc after a removal of potassium borate in the usual way. ${ }^{11)}$ As shown in Fig. 2 , the unknown sugar was separated into two components which located between raffinose and stachyose with identical $R f$ values on ppc. But the sugar corresponding to peak $D$ could not be completely separated from stachyose by this technique, even if the concentration of potassium borate was altered. Therefore, unknown sugar fraction ( $300 \mathrm{mg}$ as syrup) was at first separated from stachyose by preparative ppc and then subjected to the Dowex1 column chromatography in five portions.

\section{Oligosaccharide-I}

This oligosaccharide (I) was eluted with $\sim 8 / 1000$ M potassium borate from the Dowex-1 column, yield $130 \mathrm{mg}$ as a syrup, and had the

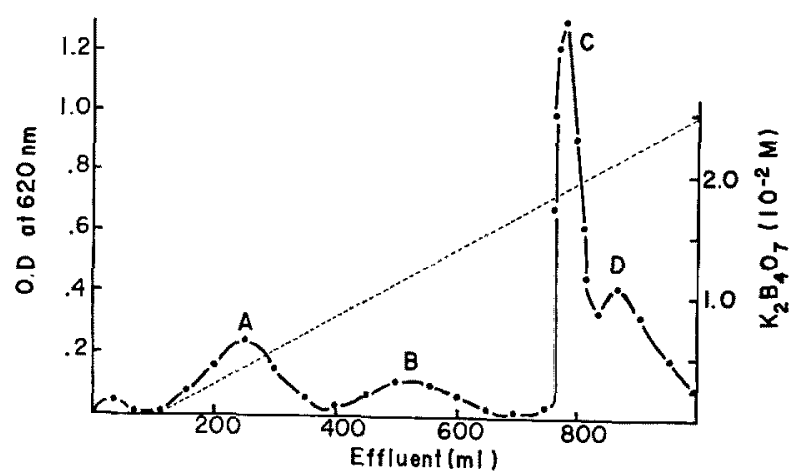

Frg. 2. Dowex-1 Column Chromatography of the Oligosaccharides.

- carbohydrate (absorbance at $620 \mathrm{~nm}$ in the Anthrone test); A, oligosaccharide-I; B, raffinose; C, stachyose; D, oligosaccharide-II. -..--, concentration of potassium borate. Conditions: Dowex -1 , X8, 200 400 mesh, borate form. Column, $12 \times 100 \mathrm{~mm}$. Eluant, $0 \sim 0.025 \mathrm{M} \mathrm{K}_{2} \mathrm{~B}_{4} \mathrm{O}_{7}$ $(0.9 \mathrm{ml} / \mathrm{min})$. 
values of $\mathrm{Mg} 0.23$ and $[\alpha]_{D}+80^{\circ}\left(c=0.2, \mathrm{H}_{2} \mathrm{O}\right)$. On acid hydrolysis, D-glucose, D-galactose and $D$-fructose were obtained. The ratio of $D$ glucose to D-galactose was almost unity under the conditions attempted. But that of Dfructose to D-galactose and/or D-glucose was not definite. This may be owing to overdegradation of D-fructose. However, I was found to contain two moles of $\mathrm{D}$-fructose residue from a result of methylation study as mentioned below.

I $(5 \mathrm{mg})$ was dissolved in a small amount of water to which was added a solution $(1 \mathrm{ml})$ of invertase $(0.5 \mathrm{mg}$, a product of Sigma Chemicals) in $0.2 \mathrm{M}$ acetate buffer ( $\mathrm{pH} 5.3$ ) and the mixture was incubated at $55^{\circ} \mathrm{C}$ for $3 \mathrm{hr}$. Sugar composition of the mixture was examined by ppc using UA as a developer after inactivation of the enzyme by addition of methanol followed by deionization with the resin IR-120 and IRA-410. Only D-fructose was detected.

I (ca. $1 \mathrm{mg}$ ) was incubated with $\alpha$-D-galactosidase (from green coffee beans, a product of boehringer mannheim) solution $(10 \mu \mathrm{l})$ at $25^{\circ} \mathrm{C}$ for $7 \mathrm{hr}$ in $0.05 \mathrm{M}$ Mcllvaine buffer ( $\mathrm{pH}$ $6.0,100 \mu 1$ ), and then submitted to ppc analysis. Only D-galactose was detected on $\mathrm{ppc}$ developed with AHP.

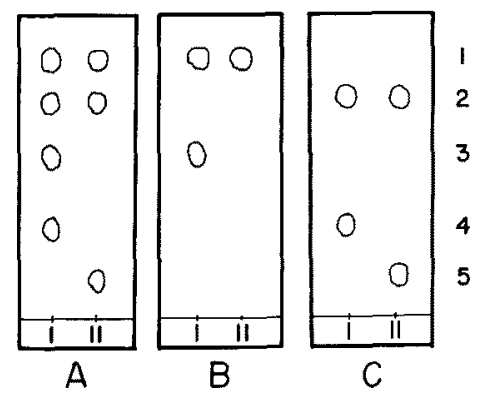

FIG. 3. Facsimiled Thin-layer Chromatograms of the Hydrolyzates of the Methylated Oligosaccharide-I and II.

$1,1,3,4,6$-tetra- $O$-methyl-D-fructose ; $2,2,3,4,6$ tetra- $O$-methyl-D-galactose; 3, 3, 4, 6-tri- $O$-methyl-Dfructose; 4, 2, 3, 4-tri- $O$-methyl-D-glucose; $5,2,3$-di$O$-methyl-D-glucose.

Solvent; $\mathrm{CHCl}_{3}: \mathrm{CH}_{3} \mathrm{OH}(25: 1)$. Spray reagent; $50 \% \mathrm{H}_{2} \mathrm{SO}_{4}(\mathrm{~A})$, urea-acid (B) and aniline hydrogen phthalate (C).
I (30 mg) was methylated by the Hakomori method, ${ }^{12}$ and the methylated product was hydrolyzed with $1 \mathrm{~N} \mathrm{HCl}$ at $100^{\circ} \mathrm{C}$ for $3 \mathrm{hr}$. The hydrolyzate was analyzed with tlc using $50 \%$ sulfuric acid, AHP and UA as developer, respectively. The results are shown in Fig. 3. A part of the hydrolyzate was treated with sodium borohydride and then acetylated with acetic anhydride and pyridine in the usual way. Alditol acetates thus obtained were analyzed $^{13}$ by the combined GC-MS. The results are shown in Figs. 4 and 5. From these findings, oligosaccharide-I was established as $O$ - $\alpha$-D-galactopyranosyl- $(1 \rightarrow 6)-O$ - $\alpha$-D-glucopyranosyl- $(1 \rightarrow 2)[O-\beta$-D-fructofuranosyl- $(2 \rightarrow$ 1)]- $\beta$-D-fructofuranoside.

\section{Oligosaccharide-II}

This oligosaccharide (II) was eluted with $\sim 20 / 1000 \mathrm{M}$ potassium borate from the Dowex-1 column, yield $170 \mathrm{mg}$ as a syrup, and gave the value of $\mathrm{Mg} 0.46$ and $[\alpha]_{\mathrm{D}}+160^{\circ}$ ( $c=0.2, \mathrm{H}_{2} \mathrm{O}$ ). On acid hydrolysis, D-glucose, D-galactose and D-fructose were obtained. The ratio of $\mathrm{D}$-glucose and $\mathrm{D}$-galactose was found to be $1: 2$. But those of D-fructose to the other sugars were similarly indefinite. However, one mole of D-fructose was found to be contained in this oligosaccharide by methylation study. This $\mathrm{D}$-fructosyl group was eliminated when incubated with the invertase under the same conditions as mentioned above.

When incubated with $\alpha$-D-galactosidase under the same conditions as mentioned above, II yielded D-galactose and sucrose, as revealed by $\mathrm{ppc}$.

Methylation of II followed by acid hydrolysis gave a mixture of partially methylated monosaccharides (Fig. 3), which were subjected to preparative tlc. Tetra- $O$-methyl-D-galactose isolated was refluxed with ethanol containing aniline and a trace of hydrochloric acid to yield a 2, 3, 4, 6-tetra- $O$-methyl- $\mathrm{N}$-phenylgalactosyl amine $e^{14}$; mp $192 \sim 194^{\circ} \mathrm{C}$ after recrystallization from ethanol. Other methylated monosaccharides could not be identified as any crystalline derivatives because of their small quantities. 


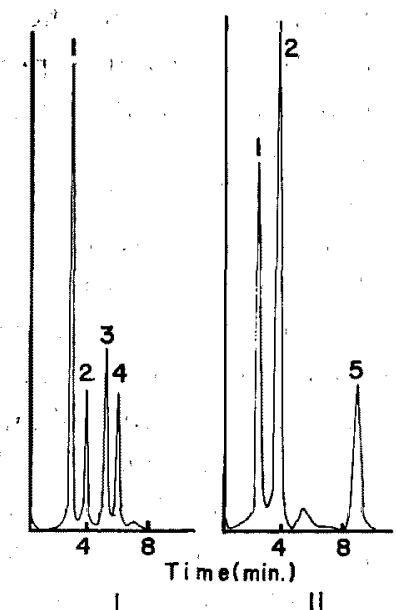

Frg. 4. Gas 'Chromatographies of the Partially Methylated Alditol Acetates Derived from Oligosaccharide-I and II.

The numbering of alditol acetate-peaks in the Figure corresponds to that in Fig. 3.

Conditions: column, $2 \%$ OV-1 $(0.3 \times 100 \mathrm{~cm})$; column temp., $160^{\circ} \mathrm{C}$; carrier, $\mathrm{He}\left(0.8 \mathrm{~kg} / \mathrm{cm}^{2}\right)$; detector, mass spectrometer.
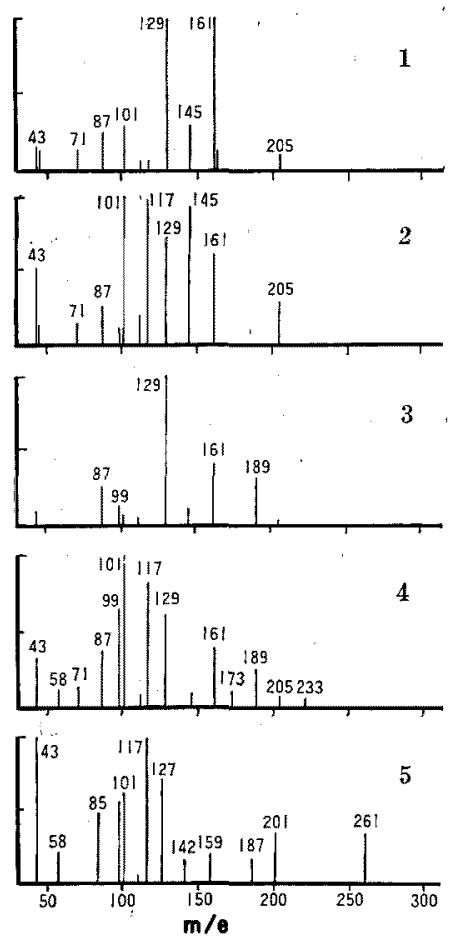

Frg. 5. Mass-Spectra of the Alditol Acetates. The numbering of the spectrum corresponds to that of the peak in Fig. 4.

Conditions: ionization voltage, $20 \mathrm{eV}$; ion source temp., $220^{\circ} \mathrm{C}$.
A part of the partially methylated monosaccharides were converted to corresponding alditol acetates and then subjected to the $\mathrm{GC}$ MS analysis. The results are shown in Figs. 4 and 5 . From these findings, oligosaccharideII was established as $O$ - $\alpha$-D-galactopyranosyl$(1 \rightarrow 6)[O-\alpha$-D-galactopyranosyl-( $(1 \rightarrow 4)]-O-\alpha-\mathrm{D}$ glucopyranosyl-( $1 \rightarrow 2)-\beta$-D-fructofuranoside.

\section{DISCUSSION}

Two kinds of new tetrasaccharide (I and II) were isolated from cottonseed meal and their structures were established, though attempts to crystallize the oligosaccharides from various solvent were not successful. They were eluted from a carbon column with almost the same retention volumes and located between raffinose and stachyose on ppc with identical $R f$ values. However, they could be separated from each other on the Dowex-1 (borate form) column chromatography and paper electrophoresis using a borate buffer.

Digestion of the oligosaccharides with invertase followed by paper chromatographic examination revealed that $\mathrm{D}$-fructose residues in the molecules should be present in the form of $\beta$-D-fructofuranoside and not substituted by other monosaccharides than $\beta$-D-fructofuranose. Detection of $\mathrm{D}$-galactose in the hydrolyzate by $\alpha$-D-galactosidase qualified D-galactosyl residue in the molecules to be a $\alpha$-Dpyranose form and not to be substituted. These results, except configuration of glycosidic linkages, were also supported by the identification of $2,3,4,6$-tera- $O$-methyl-Dgalactose and 1,3,4,6-tera- and 3,4,6-tri$O$-methyl-D-fructose on methylation study. Identification of $1,2,5$-tri- $O$-acetyl-3, 4, 6tri- $O$-methyl-sorbitol derived from 3,4,6-tri$O$-methyl-D-fructose at first confused the cumulative results on $\mathbf{I}$; this compound can be also derived from 2-O-substituted D-glucose. But this came to a perfect understanding when the hydrolyzate of methylated I was analyzed on tlc developed with a specific reagent for ketose, UA and for aldose, AHP; two spots corresponding to $1,3,4,6$-tetra- and 3, 4, 6-tri- 0 - 

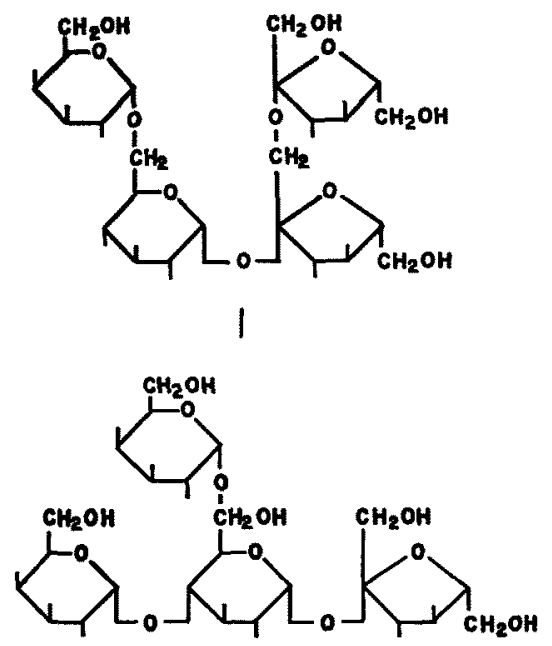

II

Fig. 6. Structures of Oligosaccharide-I and II. I, $O-\alpha-\mathrm{D}$-galactopyranosyl- $(1 \rightarrow 6)-O-\alpha-\mathrm{D}$-glucopyranosyl- $(1 \rightarrow 2) \quad[O-\beta$ - D-fructofuranosyl - $(2 \rightarrow 1)]-\beta-\mathrm{D}$ fructofuranoside; II, $O-\alpha-\mathrm{D}$-galactopyranosyl- $(1 \rightarrow 6)$ $[O$ - $\alpha$-D-galactopyranosyl- $(1 \rightarrow 4)]-O$ - $\alpha$-D-glucopyranosyl-(1 $\rightarrow 2)-\beta$-D-fructofuranoside.

methyl-D-fructose were detected with UA, and two spots corresponding to 2,3,4,6-tetra- $O$ methyl-D-galactose and 2,3,4-tri- $O$-methylD-glucose were detected with AHP.

Many galactosyl- and fructosylsucroses have been found in plant-tissues, and some of their structures were related to the transferase properties of carbohydrase in the tissues. It is well-known ${ }^{15}$ ) that invertases from planttissues transfer D-fructosyl residue from one sucrose molecule to $\mathrm{C}-1$ of $\mathrm{D}$-fructose and/or C- 6 of D-glucose residues of another sucrose molecule to form isokestose and/or neokestose, but do not form kestose in which $\mathrm{D}$-fructose joined through $\mathrm{C}-6$ of $\mathrm{D}$-fructose residue of sucrose molecule. In view of the transferase properties of plant invertase, oligosaccharideI might be formed by the action of invertase in the seed, in which raffinose, instead of sucrose, is an acceptor of D-fructosyl group.

On the other hand, galactosylsucroses such as raffinose and planteose are reported to be synthesized by the transferase action of $\alpha$-Dgalactosidase. Some studies ${ }^{16}$ ) on properties of $\alpha$-D-galactosidase from plant suggest that the enzyme transfers D-galactosyl residue from such galactosyl donors as galactinol and raffinose to primary alcohol group of hexoses and some oligosaccharides. However, transgalactosylation to $\mathrm{C}-4$ of hexoses by the enzyme has not been reported as yet. In addition, $4^{G}-\alpha$-D-galactosylsucrose has not yet been recognized in nature. Therefore, isolation of oligosaccharide-II containing such glycosidic linkage will give some information on the transferase properties of $\alpha$-D-galactosidase in cottonseed, if the sugar is formed by the action of such enzyme.

\section{REFERENCES}

1) R. W. Bailey, "Oligosaccharides," Pergamon Press Ltd., London, 1965, p. 108 112.

2) D. French, D. W. Knapp and J. H. Pazur, J. Am. Chem. Soc., 72, 5150 (1950).

3) R. L. Whistler and J. L. Hickson, Anal. Chem., 27, 1514 (1955).

4) K. Aso and S. Hamada, J. Ferment. Technol., 33, 45 (1959).

5) S. M. Partridge, Nature, 164, 443 (1949).

6) G. Zweig and J. Sherma, "Handbook of Chromatography," Vol. II, CRC Press, Cleveland, Ohio, p. 130.

7) W. E. Trevelyan, D. P. Procter and J. S. Harrison, Nature, 166, 444 (1950).

8) N. Dubois, J. K. Hamilton, K. A. Gillis, P. A. Rebers and F. Smith, Anal. Chem., 28, 350 (1956).

9) G. R. Noggle and L. P. Zill, Arch. Biochem. Biophys., 41, 21 (1952).

10) R. J. Dimler, W. C. Shaefer, C. S. Wise and C. E. Rist, Anal. Chem., 24, 1411 (1952).

11) L. P. Zill, J. X. Khym and G. M. Cheniac, J. Am. Chem. Soc., 75, 1339 (1953).

12) S. Hakomori, J. Biochem., 55, 205 (1964).

13) H. Björndal, B. Lindberg and S. Svensson, Carbohydr. Res, , 5, 433 (1967).

14) L. Hough and J. K. N. Jones, J. Chem. Soc., 1950, 1199.

15) P. J. Allen and J. S. D. Bacon, Biochem. J., 63, 200 (1956).

16) P. M. Dey and J. B. Pridham, Adv. Enzymol., 36, 91 (1972). 